\title{
ISOLOGY THEORY
}

\author{
BY \\ ARTHUR H. COPELAND, JR.(1)
}

Introduction. The successes of algebraic methods in homotopy theory raise the hope of a similar success in the closely related field of isotopy theory. In particular, one may hope to find analogs of the homology and cohomology functors, to find a functor whose ability to distinguish isotopy equivalences from the other embeddings matches the homotopy functor's ability to distinguish homotopy equivalences from the other maps, and eventually to find classifying systems analogous to the Postnikov systems. Some of the goals of such a search would be the classification up to isotopies of the embeddings of a given space in another when the spaces are of a sufficiently simple nature, the classification into isotopy types of spaces from a suitable category, and the determination of when a given embedding of a subset of a space $X$ into a space $Y$ has an extension, over $X$, which is also an embedding. Note that the item mentioned last subsumes the problem of deciding when a given space $X$ may be embedded in a space $Y$.

The first step in the direction of finding functors of the types described above was taken by S.-T. Hu [3]. His isotopy functors, although basically different from homology functors, have this similarity: they satisfy modified EilenbergSteenrod axioms, excepting the Excision Axiom. This gives them an advantage in strength, in the sense that they may distinguish many isotopy classes, but a disadvantage in analysis. That is, there is no obvious way, as there would be if the Excision Axiom were present, of dividing up a given space into elementary parts, studying the action of the functor on the parts and reassembling these results to obtain the value of the functor on the space.

The present paper studies functors, called isology theories, which also satisfy the Excision Axiom. That is, an isology theory is a functor $\left\{T_{p}\right\}$, on a category $\Gamma$ of embeddings of topological pairs, which has values in the category of homomorphisms of abelian groups and which satisfies slightly modified EilenbergSteenrod axioms. Roughly speaking, the modification consists of replacing the word "homotopy" in Axiom Five by the word "isotopy."

Note that every homology theory is an isology theory. However, it is found that if $\Gamma$ is the category of embeddings of (finitely) triangulable pairs, then there exist isology theories on $\Gamma$ other than the homology theories. Indeed, it is possible to find a nontrivial theory with a trivial coefficient group. More important, these

Received by the editors May 22, 1961 and, in revised form, May 14, 1962.

(1) Sponsored by the U. S. Army Research Office (Durham). 
theories distinguish the isotopy types of certain contractible spaces. The techniques used in the construction of these theories are not powerful enough to provide theories which distinguish all of the contractible spaces. However, if $\Gamma$ is the category of embeddings of 1-dimensional triangulable pairs, then a different construction produces isology theories which distinguish between the contractible spaces. Furthermore, in this category a theorem holds which can be summarized roughly as follows: an isology theory which distinguishes the isotopy types of contractible spaces from each other must distinguish the isotopy equivalences between spaces from the other embeddings. Specifically, the isotopy equivalences are the only embeddings which induce isomorphisms.

The other area investigated in this paper is the determination of the values of a given isology functor on triangulable spaces and pairs. These determinations are expressed in terms of the value of the functor on certain of the contractible spaces. If the functor satisfies a vastly strengthened form of the Dimension Axiom, then there is an algorism for computing the isology groups of triangulable pairs. Otherwise, the isology groups are related to a family of a such algorisms by means of a spectral sequence.

The author wishes to express his gratitude to K. R. Mount, A. I. Weinzweig and the referee for their many valuable suggestions.

1. Notation and conventions. Let $\Gamma$ be a category of maps (= continuous functions) between topological pairs, and assume that $\Gamma$ satisfies the following conditions.

1. If $f$ and $g$ are maps in $\Gamma$ and if the composite $f g$ is defined, then $f g$ is in $\Gamma$.

2. If $(X, A)$ is an object in $\Gamma$, then the objects and inclusion maps in the lattice of $(X, A)$ are in $\Gamma$.

3. If $f:(X, A) \rightarrow(Y, B)$ is a map in $\Gamma$, then the induced maps of the lattice of $(X, A)$ into the lattice of $(Y, B)$ are in $\Gamma$.

The lattice of a pair $(X, A)$ is the system of all inclusion maps among the pairs $(\square, \square),(A, \square),(X, \square),(A, A),(X, A)$ and $(X, X)$, where $\square$ denotes the empty set. Note that a category for homology is a category of the type postulated here, but not conversely.

A homotopy in $\Gamma$ is a one-parameter family

$$
f_{t}:(X, A) \rightarrow(Y, B), \quad t \in I,
$$

of maps in $\Gamma$ such that the map $(x, t) \rightarrow f_{t}(x)$ is continuous on $X \times I$ into $Y$. Two maps $f, g \in \Gamma$ are homotopic in $\Gamma$ (written $f \sim g$ ) if there is a homotopy $f_{t}$ in $\Gamma$ such that $f_{0}=f$ and $f_{1}=g$. In case $\Gamma$ contains all maps between its pairs, the notion of homotopy just defined coincides with the usual notion.

An embedding $f:(X, A) \rightarrow(Y, B)$ of the topological pair $(X, A)$ in the pair $(Y, B)$ is a map whose restriction to $X$ is a homeomorphism of $X$ onto a subset of $Y$. Unless explicitly stated otherwise, all categories are to be categories of 
embeddings. Thus a homotopy in $\Gamma$ becomes an isotopy, and homotopic maps are isotopic.

Recall that the first six of the seven Eilenberg-Steenrod Axioms for Homology [2] are:

(1) (identity) $)_{*}=$ identity.

(2) $(f g)_{*}=f_{*} g_{*}$.

(3) $f_{*}=(f A)_{*}$.

(4) Exactness.

(5) Homotopy. If $f \sim g$, then $f_{*}=g_{*}$.

(6) Excision.

An isology theory on $\Gamma$ is any functor satisfying these six axioms, with the modification that, in Axiom Five, $f \sim g$ is to be interpreted in the sense just described. Some caution needs to be exercised relative to this modification, for the map $(x, t) \rightarrow f_{t}(x)$ need not be in $\Gamma$. In particular, the condition listed in [2] as Axiom 5': If $(X, A)$ is admissible and $g_{0}, g_{1}:(X, A) \rightarrow(X, A) \times I$ are defined by $g_{0}(x)=(x, 0), g_{1}(x)=(x, 1)$, then $g_{0} *=g_{1} *$, is not equivalent to Axiom 5 .

One observes immediately that any homology theory restricted to $\Gamma$ is an isology theory. Customarily, we shall use $T_{p}(X, A)$ to denote the $p$ th isology group of $(X, A) \in \Gamma$, reserving $H_{p}(X, A ; G)$ for the $p$ th singular homology group of $(X, A)$ with coefficients in the abelian group $G$.

A second observation is that the Dimension Axiom has been omitted. This is because its original form is too weak to be useful in the present context. An illustration of this weakness is given in $\S 3$, where it is shown that the value of the isology functor on a one-point space has almost no bearing on its value elsewhere. One way around this difficulty is to replace the Dimension Axiom by one of a family of much stronger Dimension Postulates $\left\{a_{n}\right\}$, described below.

A 0 -cone is defined to be a single point. For $n>0$, an n-cone is a homeomorph of the join of any finite $(n-1)$-complex $K$ with a point. That is, it is the quotient space obtained from $K \times I$ by collapsing $K \times\{1\}$ to a point. The image of $K \times\{1\}$ is called the apex of the cone. Note that a space $A$ is an $n$-cone if and only if there are an $n$-complex $L$ and a vertex $v$ in $L$ such that $A$ is homeomorphic to the closed star of $v$ in $L$. A space is called a cone if it is an $n$-cone for some nonnegative integer $n$.

Let $a_{0}, a_{1}, a_{2}, \cdots$ be a monotone nondecreasing sequence on nonnegative integers.

Dimension Postulate $\left\{a_{n}\right\}$. If $A \in \Gamma$ is an $n$-cone, then $T_{p}(A)=0$ except possibly when $0 \leqq p \leqq a_{n}$.

Note that a homology theory satisfies the Dimension Postulate $\{0\}$. One more postulate has proved useful, especially in the computation of the isology groups of pairs.

MONOMOR PHISM Postulate. If $A$ and $B$ are cones with apexes $a$ and $b$, and if $f:(A, a) \rightarrow(B, b)$ is in $\Gamma$, then 


$$
T_{p}(f): T_{p}(A) \rightarrow T_{p}(b)
$$

is a monomorphism for all $p$.

A final observation is that much of Chapter I of Eilenberg-Steenrod [2] holds, provided the word "homotopy" is replaced by "isotopy." In particular,

1. An isotopy equivalence induces isomorphisms.

2. The isology sequence of a triple is exact.

3. The Meyer-Vietoris sequence of a proper triad is exact.

The $n$-fold product of the unit interval $[0,1]=I$ with itself is the $n$-cube $I^{n}$, and $\Delta_{n}$ is the standard $n$-simplex.

2. A Strong Excision Axiom. The delicacy with which the Excision Axiom must be handled is one of the more surprising features of the present study. Indeed, the whole theory collapses to ordinary homology theory when another, quite familiar form of the Excision Axiom is used.

Strong Excision Axiom. If $(X, A)$ is a triangulable pair and if $U \subset A$ is an open subset of $X$ such that $(X \backslash U, A \backslash U)$ is triangulable, then the inclusion map induces an isomorphism

$$
T_{p}(X \backslash U, A \backslash U) \approx T_{p}(X, A) .
$$

Let us identify the $(q-1)$-cube $I^{q-1}$ with the subset $I^{q-1} \times\{0\}$ of $I^{q}$.

Lemma. If $T_{p}$ is an isology theory satisfying the Strong Excision Axiom, then

$$
T_{p}\left(I^{q}, I^{q-1}\right)=0 \quad \text { for all } p, q .
$$

Proof. The inclusion map

$$
I^{q-1} \times[-1,0] \rightarrow I^{q-1} \times[-1,1]
$$

is an isotopy equivalence, and the inclusion map

$$
\left(I^{q}, I^{q-1}\right) \rightarrow\left(I^{q-1} \times[-1,1], I^{q-1} \times[-1,0]\right)
$$

satisfies the hypothesis of the Strong Excision Axiom. Thus

$$
T_{p}\left(I^{q}, I^{q-1}\right) \approx T_{p}\left(I^{q-1} \times[-1,1], I^{q-1} \times[-1,0]\right)=0 .
$$

THEOREM. If $\left\{T_{p}\right\}$ is an isology theory on the category $\Gamma_{0}$ of embeddings of finitely triangulable pairs and if this theory satisfies the Dimension Axiom and the Strong Excision Axiom, then

$$
T_{p}(X, A) \approx H_{p}\left(X, A ; T_{0}\left(I^{0}\right)\right)
$$

for all $(X, A) \in \Gamma_{0}$ and for all integers $p$. 
The proof is simply Chapter III of Eilenberg-Steenrod [2] with two modifications: Theorems 2.1 and 2.2 on pp. 76 and 77 are versions of the Strong Excision Axiom, and Lemma 3.1 on p. 78 follows from the lemma just proved in the present section.

3. Nontrivial isology theories. In this section, it is shown that the category $\Gamma_{0}$ of embeddings of triangulable pairs admits isology theories other than the standard homology theories. These theories may be adjusted to fit any given dimension postulate. In addition, there are theories $\left\{T_{p}\right\}$ such that $T_{p}\left(\Delta_{0}\right)=0$, for all integers $p$, but which are not trivial. The main theorem of the section is as follows.

THEOREM 3.1. Let $\left\{G_{p q}: p, q\right.$ integers, $\left.q \geqq 0\right\}$ be a family of abelian groups. There exists an isology theory $\left\{T_{p}\right\}$ on $\Gamma_{0}$ such that

$$
T_{p}\left(\Delta_{q}\right)=G_{p q} \text { for all } p, q .
$$

Before embarking on the proof of this theorem, let us examine some of its consequences. In the category of all maps of triangulable pairs, a homology theory is determined by $T_{0}\left(\Delta_{0}\right)$. In particular, if $T_{0}\left(\Delta_{0}\right)=0$, the theory is trivial on all pairs. I. M. James and J. H. C. Whitehead [4] have shown that if the category on which a homology theory operates is "too large," then uniqueness fails and there may be nontrivial theories with $T_{0}\left(\Delta_{0}\right)=0$. Theorem 3.1 of the present paper may be interpreted as saying that if the category is "too small" the uniqueness fails again and there may be nontrivial theories with $T_{0}\left(\Delta_{0}\right)=0$.

The isology theories given by Theorem 3.1 may distinguish between various contractible spaces. In particular, there are theories which have

$$
T_{p}(A)=0
$$

whenever $A$ is an (n-1)-cone, and yet have nontrivial $p$ th isology groups for certain $n$-cones. This consideration has been important in formulating the $\mathrm{Di}$ mension Postulates.

If $X$ is a topological space and if $n \geqq 0$ is an integer, let $U_{n} X$ be the union of all closed topological $n$-cells contained in $X$. That is, $U_{n} X$ is the union of all homeomorphic images in $X$ of the $n$-cube $I^{n}$. Note that if $X$ and $Y$ are homeomorphic spaces, then $U_{n} X$ and $U_{n} Y$ are homeomorphic. Also, $U_{0} X=X$ and $U_{m} X \supset U_{n} X$ whenever $m \leqq n$.

If $m$ and $n$ are integers with $0 \leqq m \leqq n$ and if $(X, A)$ is a topological pair, let

$$
U_{m n}(X, A)=\left(U_{m} X, U_{m} A \cup U_{n} X\right) .
$$

If $f: X \rightarrow Y$ is an embedding, then $f\left[U_{n} X\right] \subset U_{n} Y$ for each integer $n$. Thus an embedding $f:(X, A) \rightarrow(Y, B)$ induces, by restrictions, an embedding

$$
U_{m n} f: U_{m n}(X, A) \rightarrow U_{m n}(Y, B) .
$$


The following two lemmas are immediate consequences of these definitions.

LEMMA 3.2. Let $\mathscr{T}$ be a triangulation of the space $X$. Then $U_{n} X$ is the union of all closed simplexes of $\mathscr{T}$ whose dimension is at least $n$.

LEMMA 3.3. The functor $U_{m n}$ preserves isotopies.

THEOREM 3.4. If $m, n$ and $q$ are integers with $0 \leqq m \leqq n$, and if $G$ is an abelian group, then

$$
\begin{aligned}
T_{p}(X, A) & =H_{p+q}\left(U_{m n}(X, A) ; G\right), \\
T_{p}(f) & =\left(U_{m n}(f)\right)_{*}
\end{aligned}
$$

defines an isology theory on $\Gamma_{0}$.

Proof. Axioms 1, 2 and 3 are immediate. Axiom 5 follows from Lemma 3.3. The inclusion map

$$
\left(U_{m} A, U_{n} A\right) \rightarrow\left(U_{m} A \cup U_{n} X, U_{n} X\right)
$$

induces isomorphisms in homology because ordinary homology satisfies the Strong Excision Axiom, whence the exactness of the isology sequence of the pair $(X, A)$ follows from the exactness of the homology sequence of the triple $\left(U_{m} X, U_{m} A \cup U_{n} X, U_{n} X\right)$. Only the Excision Axiom remains.

Let $(X, A)$ be an object in $\Gamma_{0}$, and let $U$ be an open set in $X$ whose closure $U^{-}$is interior to $A$ and such that $(X \backslash U, A \backslash U)$ is triangulable. There is a triangulation $\mathscr{T}$ of $(X, A)$ and an open subset $V$ of $X$ such that

(i) $U \subset V \subset V^{-}$is interior to $A$,

(ii) $(X \backslash V, A \backslash V)$ is a subcomplex of $(X, A)$, relative to $\mathscr{T}$.

Let $W=U_{m} X \backslash U_{m}(X \backslash V)$. From Lemma 3.1, it follows that

Thus,

$$
U_{m}(A \backslash V) \cup U_{n}(X \backslash V)=\left(U_{m} A \cup U_{n} X\right) \cap U_{m}(X \backslash V) .
$$

(iii) $W^{-}$is interior to $U_{m} A \cup U_{n} X$,

(iv) $U_{m}(A \backslash V) \cup U_{n}(X \backslash V)=\left(U_{m} A \cup U_{n} X\right) \backslash W$,

whence the inclusion map induces,

$$
H_{p+q}\left(U_{m n}(X \backslash V, A \backslash V) ; G\right) \approx H_{p+q}\left(U_{m n}(X, A) ; G\right) .
$$

On the other hand,

whence

$$
A \backslash V \subset A \backslash U \subset X \backslash U
$$

$$
\left(U_{m} A \cup U_{n} X\right) \backslash W \subset U_{m}(A \backslash U) \cup U_{n}(X \backslash U) .
$$

From this and (iii), it follows that $W^{\prime}$ is interior to $U_{m}(A \backslash U) \cup U_{n}(X \backslash U)$ when $W^{\prime}=W \cap\left(U_{m}(A \backslash U) \cup U_{n}(X \backslash U)\right)$. Thus the inclusion map induces 


$$
H_{p+q}\left(U_{m n}(X \backslash V, A \backslash V) ; G\right) \approx H_{p+q}\left(U_{m n}(X \backslash U, A \backslash U) ; G\right) .
$$

The Excision Axiom now follows.

The fact that $\left\{H_{q}(; G)\right\}$ satisfies the Dimension Axiom has not been used. Thus a result similar to Theorem 3.4 may be obtained using a generalized homology theory (in the sense of [5]).

Proof of Theorem 3.1. If $(X, A)$ is an object in $\Gamma_{0}$, let $T_{r}(X, A)$ be the weak direct sum,

$$
T_{r}(X, A)=\sum_{p, q} H_{r-p}\left(U_{q, q+1}(X, A) ; G_{p q}\right) .
$$

It follows easily from Theorem 3.4 that $\left\{T_{r}\right\}$ is an isology theory. If $p$ and $q$ are integers and if $q \geqq 0$, then the only nontrivial summand in $T_{p}\left(\Delta_{q}\right)$ is

$$
T_{p}\left(\Delta_{q}\right)=H_{0}\left(\Delta_{q} ; G_{p, q}\right)=G_{p, q} .
$$

Although the isology theories of this section are closely related to ordinary homology theories, they do distinguish between certain contractible spaces. For example, if $X$ and $Y$ are the 2-complexes sketched below, then $H_{0}\left(U_{2} X ; G\right) \approx G$ and $H_{0}\left(U_{2} Y ; G\right) \approx G+G$.

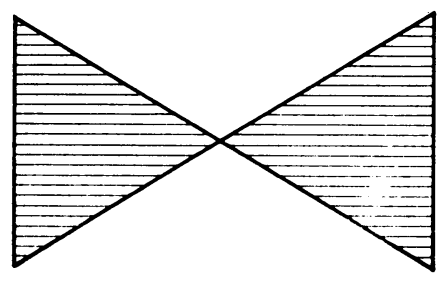

$X$

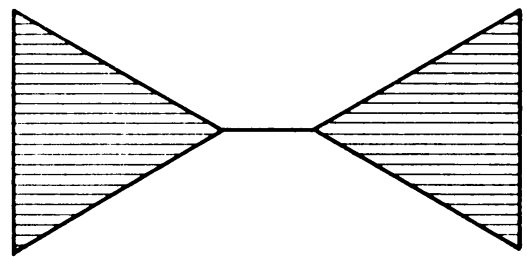

$Y$

Similar applications of this idea abound. On the other hand, these theories do not distinguish between cones of the same dimension.

THEOREM 3.5. Let $A=v * K$ be an $n$-cone and suppose $K$ contains an $(n-1)$-simplex $\sigma$. Then the inclusion map $i: v * \sigma \rightarrow A$ induces isomorphisms

$$
i_{*}: H_{p}\left(U_{q} v * \sigma, U_{r} v * \sigma\right) \approx H_{p}\left(U_{q} A, U_{r} A\right)
$$

for all $p, q$ and $r$ with $q<r$.

Proof. The four spaces $U_{q} v * \sigma, U_{r} v * \sigma, U_{q} A, U_{r} A$ are all contractible or empty, depending upon whether the subscript is $\leqq n$ or $>n$. The theorem follows easily.

In $\S 8$, a theory which distinguishes cones of the same dimension is presented. This theory acts on a very limited category, the category of 1-dimensional triangulable pairs.

4. Spectral sequences for isology. For triangulable pairs $(X, A) \in \Gamma_{0}$, the isology groups may be studied by means of a spectral sequence whose terms 
$E_{p q}^{2}$ are finitely computable in terms of the isology of cones. As a corollary, the isology groups of a triangulable space are finitely computable when the isology theory satisfies the Dimension Postulate $\{0\}$. This section is devoted to stating these results and to describing the algorisms for determining $E_{p q}^{2}$. The following three sections contain the proofs.

Let $X$ be a triangulable space with triangulation $\mathscr{T}$. The term simplex of $\mathscr{T}$ shall always be interpreted as open simplex (with the boundary removed). If $B \subset X$ is a union of simplexes of $\mathscr{T}$, let $s B$ be the union of all simplexes of having a face which lies in $B$. Note that if $B=\bar{\sigma}$ is the closure of a single simplex, then $s \bar{\sigma}$ is the open star of $\sigma$. Let us abbreviate $s \bar{\sigma}$ to $s \sigma$. If $\tau$ is a face of $\sigma$, then $s \sigma \subset s \tau$.

Let $\left\{[\sigma: \tau]_{0}\right\}$ be a system of incidence numbers for the triangulation $\mathscr{T}$. To each integer $q$ there is assigned a chain complex $C(X ; q)$ as follows. The $p$-dimensional group of the complex is the direct sum

$$
C_{p}(X ; q)=\sum\left\{T_{q}(s \sigma): \sigma \text { is a } p \text {-simplex of } \mathscr{T}\right\} .
$$

If $\tau$ is a $(p-1)$-face of the $p$-simplex $\sigma$, define the incidence homomorphism to be

$$
[\sigma: \tau]=[\sigma: \tau]_{0}(\text { inclusion })_{*}: T_{q}(s \sigma) \rightarrow T_{q}(s \tau) .
$$

The boundary homomorphism

is defined by

$$
\partial: C_{p}(X ; q) \rightarrow C_{p-1}(X ; q)
$$

$$
\partial(g)=\sum\{[\sigma: \tau](g): \tau \text { is a face of } \sigma\}
$$

when $g \in T_{q}(s \sigma)$.

If $(X, A) \in \Gamma_{0}$, we define the chain complex $C(X, A ; q)$ as follows. If the simplex $\sigma$ lies in $A$, then the star of $\sigma$ in $A$ is

$$
s_{A} \sigma=A \cap s \sigma \subset s \sigma .
$$

These inclusion maps induce a chain homomorphism

$$
i_{*}: C_{p}(A ; q) \rightarrow C_{p}(X ; q)
$$

for each $p$. Then set

$$
C_{p}(X, A ; q)=\operatorname{coker} i_{*} .
$$

The boundary homomorphism of $C(X, A ; q)$ is determined in the standard fashion.

THEOREM 4.1. If $X$ is a space with triangulation $\mathscr{T}$, then there is a spectral sequence $\left\{E_{p q}^{r}\right\}$ with

$$
E_{p q}^{2}=H_{p}(C(X ; q))
$$

whose limit terms $E_{p q}^{\infty}$ are quotients of a filtration of $T_{p}(X)$. 
Since the star of a simplex has the isotopy type of a cone, we have the following corollary.

Corollary 4.2. If $\left\{T_{p}\right\}$ satisfies the Dimension Postulate $\{0\}$, then

$$
T_{p}(X)=H_{p}(C(X ; 0)) .
$$

THEOREM 4.3. Suppose $\left\{T_{p}\right\}$ is an isology theory on $\Gamma_{0}$ satisfying the Monomorphism Postulate. If $(X, A) \in \Gamma_{0}$ has a triangulation $\mathscr{T}$, then there is a spectral sequence $\left\{E_{p q}^{r}\right\}$ with

$$
E_{p q}^{2}=H_{p}(C(X, A ; q))
$$

whose limit terms $E_{p q}^{\infty}$ are quotients of a filtration of $T_{p}(X, A)$.

Corollary 4.4. If, in addition, $\left\{T_{i}\right\}$ satisfies the Dimension Postulate $\{0\}$, then

$$
T_{p}(X, A)=H_{p}(C(X, A ; 0)) .
$$

Note that in Theorems 4.1 and $4.3, E_{p q}^{2}=0$ for $p$ negative and for $p$ greater than the dimension of $X$. This insures that $E_{p q}^{r}=E_{p q}^{\infty}$ for $r$ sufficiently large.

5. Isology of cones. If $\mathscr{T}$ is a triangulation of a space or a pair, let $\mathscr{T}^{\prime}$ denote the first barycentric subdivision of $\mathscr{T}$. If $B$ is a union of simplexes in $\mathscr{T}^{\prime}$, let $s^{\prime} B$ be the union of all the simplexes of $\mathscr{T}^{\prime}$ having a face lying in $B$. Ordinarily, $K^{-}$will be used to denote the closure of the subset $K$, but if the resulting expression is awkward, $\mathrm{Cl}(\mathrm{K})$ will be used instead.

If $K$ and $L$ are topological spaces then the join $K * L$ is the quotient space obtained from $K \times L \times I$ by making the following identifications:

$$
\begin{aligned}
& (x, y, 0) \equiv\left(x^{\prime}, y, 0\right), \\
& (x, y, 1) \equiv\left(x, y^{\prime}, 1\right)
\end{aligned}
$$

for all $x, x^{\prime} \in K$ and $y, y^{\prime} \in L$. A triangulation determines a linear structure on each simplex $\tau$, so that if $x, y \in \tau$ and if $t \in I$, then $t x+(1-t) y$ is a uniquely determined point of $\tau$. Thus if $\sigma$ is a simplex and if

$$
K \subset \bar{\sigma}, \quad L \subset \overline{s \sigma}
$$

are subsets, then the function

$$
(x, y, t) \rightarrow t x+(1-t) y, \quad x \in K, y \in L, t \in I,
$$

defines a map, the standard map, of $K * L$ into $\overline{s \sigma}$.

LEMMA 5.1. For each simplex $\sigma \in \mathscr{T}$ there is a complex $K=K(\sigma)$ in $\mathscr{T}^{\prime}$ such that

(i) $K \subset s \sigma$,

(ii) the standard map sends $\bar{\sigma} * K$ homeomorphically onto $\mathrm{Cl}\left(s^{\prime} \sigma\right)$. 
Proof. The complex $K$ is defined to be the union of all simplexes in $\mathscr{T}^{\prime}$ spanned by barycenters

$$
b \tau_{0}, b \tau_{1}, \cdots, b \tau_{q}
$$

of simplexes $\tau_{0}, \tau_{1}, \cdots, \tau_{q}$ in $\mathscr{T}$ such that

$$
\bar{\sigma} \subset \bar{\tau}_{0} \subset \bar{\tau}_{1} \subset \cdots \subset \bar{\tau}_{q} .
$$

Since each simplex $\tau_{r}$ lies in $s \sigma$, so must $K$. Clearly the standard map $g_{K}$ carries $\bar{\sigma} * K$ onto $\mathrm{Cl}\left(s^{\prime} \sigma\right)$. It remains to show that $g_{K}$ is a homeomorphism. If $\tau$ is a simplex of $\mathscr{T}$ having $\sigma$ as a face, let $\rho \tau$ be the face of $\tau$ opposite to $\sigma$. Then

$$
L=\bigcup\{\rho \tau: \sigma \text { is a face of } \tau\}
$$

is a complex in $\mathscr{T}, L \subset \overline{s \sigma}$, and Alexander shows that the standard map $g_{L}: \bar{\sigma} * L \rightarrow \overline{s \sigma}$ is a homeomorphism onto [1]. Let $L^{\prime}$ be the first barycentric subdivision of $L$ and let $f: K \rightarrow L^{\prime}$ be defined by the vertex map

$$
f(b \tau)=b \rho \tau \quad \text { when } \sigma \subset \bar{\tau} .
$$

Then $f$ is a homeomorphism, it induces a homeomorphism

$$
f_{1}: \bar{\sigma} * K \rightarrow \bar{\sigma} * L,
$$

and it relates the images under $g_{K}$ and $g_{L}$ as follows. The barycenters $b \sigma, b \tau$ and $b \rho \tau$ are collinear. Thus $f$ is simply a radial expansion from the point $b \sigma$, and induces a homeomorphism $f_{2}$ of $\mathrm{Cl}\left(s^{\prime} \sigma\right)$ onto $\mathrm{Cl}(s \sigma)$. It follows that

$$
g_{K}=f_{2}^{-1} g_{L} f_{1}
$$

is a homeomorphism.

The author is indebted to A. I. Weinzwieg for the foregoing proof.

Let $\sigma$ be a $p$-simplex of the triangulation $\mathscr{T}$. A flag on $\sigma$ is a sequence $\sigma_{0}, \sigma_{1}, \cdots, \sigma_{p}=\sigma$ of faces such that

(i) $\sigma_{i-1}$ is a face of $\sigma_{i}$ for $i=1,2, \cdots, p$,

(ii) $\operatorname{dim} \sigma_{i}=i$ for $0,1,2, \cdots, p$.

Let $\dot{s}^{\prime} \sigma=s^{\prime} \sigma \cap s^{\prime} \dot{\sigma}$.

LEMMA 5.2. To each flag there is assigned an isomorphism

$$
\phi: T_{p+q}\left(s^{\prime} \sigma, \dot{s}^{\prime} \sigma\right) \rightarrow T_{q}(s \sigma) .
$$

Proof. Let $\sigma_{0}, \sigma_{1}, \cdots, \sigma_{p}=\sigma$ be the given flag. For $r=0,1,2, \cdots, p$, let $v_{r}$ be the vertex of $\sigma_{r}$ opposite $\sigma_{r-1}$ and let $\tau_{r}$ be the face of $\sigma$ spanned by $v_{r+1}, v_{r+2}, \cdots, v_{p}$. Note that $\tau_{r}$ has dimension $p-r-1$ and that $\bar{\sigma}_{r} * \bar{\tau}_{r}=\bar{\sigma}$. Let $K=K(\sigma)$ and define the subsets

$$
\begin{aligned}
& U_{r}=s^{\prime} \sigma \backslash\left(b \sigma_{r} * \tau_{r} * K\right), \\
& V_{r}=s^{\prime} \sigma !\left(b \sigma_{r-1} * b \tau_{r} * \tau_{r} * K\right) .
\end{aligned}
$$


Note that $U_{p}=\dot{s}^{\prime} \sigma$.

The map on $\sigma_{r} * v_{r+1} \supset \sigma_{r+1}$ which carries $v_{r+1}$ into $b \sigma_{r+1}$, and leaves the rest of the vertices fixed, induces an embedding

$$
f_{r}:\left(s^{\prime} \sigma, U_{r}\right) \rightarrow\left(U_{r+1}, V_{r+1}\right)
$$

If $M=\bar{\sigma}_{r} * b \sigma_{r+1} * \tau_{r+1} * K$, then this embedding may be factored

$$
\left(s^{\prime} \sigma, U_{r}\right) \rightarrow\left(U_{r+1} \cap M, V_{r+1} \cap M\right) \rightarrow\left(U_{r+1}, V_{r+1}\right)
$$

where the first map is an isotopy equivalence and the second is an excision. Thus

$$
f_{r *}: T_{s}\left(s^{\prime} \sigma, U_{r}\right) \rightarrow T_{s}\left(U_{r+1}, V_{r+1}\right)
$$

is an isomorphism for all $r$ and $s$. Now define

$$
\phi=i_{*}\left(f_{0 *}\right)^{-1} \partial\left(f_{1 *}\right)^{-1} \partial \cdots \partial\left(f_{p-1 *}\right)^{-1} \partial,
$$

where the homomorphisms are as indicated in the diagram below.

$$
\begin{gathered}
T_{p+q}\left(s^{\prime} \sigma, \dot{s}^{\prime} \sigma\right)=T_{p+q}\left(s^{\prime} \sigma, U_{p}\right) \\
T_{p+q-1}\left(s^{\prime} \sigma, U_{p-1}\right)-\frac{\partial}{f_{p-1 *}} T_{p+q-1}
\end{gathered}
$$

The inclusion map $i: s^{\prime} \sigma \rightarrow s \sigma$ is an isotopy equivalence, whence $i_{*}$ is an isomorphism. The inclusion map

$$
\sigma_{r} \backslash\left(b \sigma_{r-1} * b \sigma_{r}\right) \rightarrow \sigma_{r}
$$

is an isotopy equivalence, whence the inclusion map

$$
V_{r} \rightarrow s^{\prime} \sigma
$$

is an isotopy equivalence. It follows that $T_{s}\left(s^{\prime} \sigma, V_{r}\right)=0$ for all $r, s$ whence each $\partial$ is an isomorphism. Thus the composite $\phi$ is an isomorphism.

A permutation $\pi$ of the vertices of $\sigma$ induces a homeomorphism of $s^{\prime} \sigma$ on itself. Given two flags on $\sigma$ there exists a permutation $\pi$ which carries the first flag onto the second. 
LEMMA 5.3. If $\sigma_{0}^{0}, \sigma_{1}^{0}, \cdots, \sigma_{p}^{0}=\sigma$ and $\sigma_{0}^{1}, \sigma_{1}^{1}, \cdots, \sigma_{p}^{1}=\sigma$ are two flags on the p-simplex $\sigma$, then the corresponding isomorphisms $\phi^{0}$ and $\phi^{1}$ satisfy

$$
\phi^{0}=\operatorname{sgn}(\pi) \phi^{1},
$$

where $\pi$ is the permutation relating the two flags.

Proof. It suffices to show that if the two flags differ on exactly one face $\sigma_{r}^{0} \neq \sigma_{r}^{1}$, then $\phi^{0}=-\phi^{1}$. Let

$$
W=s^{\prime} \sigma \backslash\left(b \sigma_{r-1} *\left(b \sigma_{r}^{0} \cup b \sigma_{r}^{1}\right) * b \sigma_{r+1} * \tau_{r-1} * K\right) .
$$

Note that

$$
f_{r-1}^{0} f_{r}^{0} \sim f_{r-1}^{1} f_{r}^{1}:\left(s^{\prime} \sigma, U_{r-1}\right) \rightarrow\left(V_{r+1}^{0} \cap V_{r+1}^{1}, W\right) .
$$

The notation follows the scheme in Lemma 5.2. The superscripts 0 and 1 are omitted from a symbol whenever there is no difference between the sets or maps seemingly differentiated by the superscripts. If $i^{0}$ and $i^{1}$ are inclusion maps and if $g^{0}, g^{1}$ are induced by $f_{r}^{0}$ and $f_{r}^{1}$, respectively, then the diagram below is commutative.

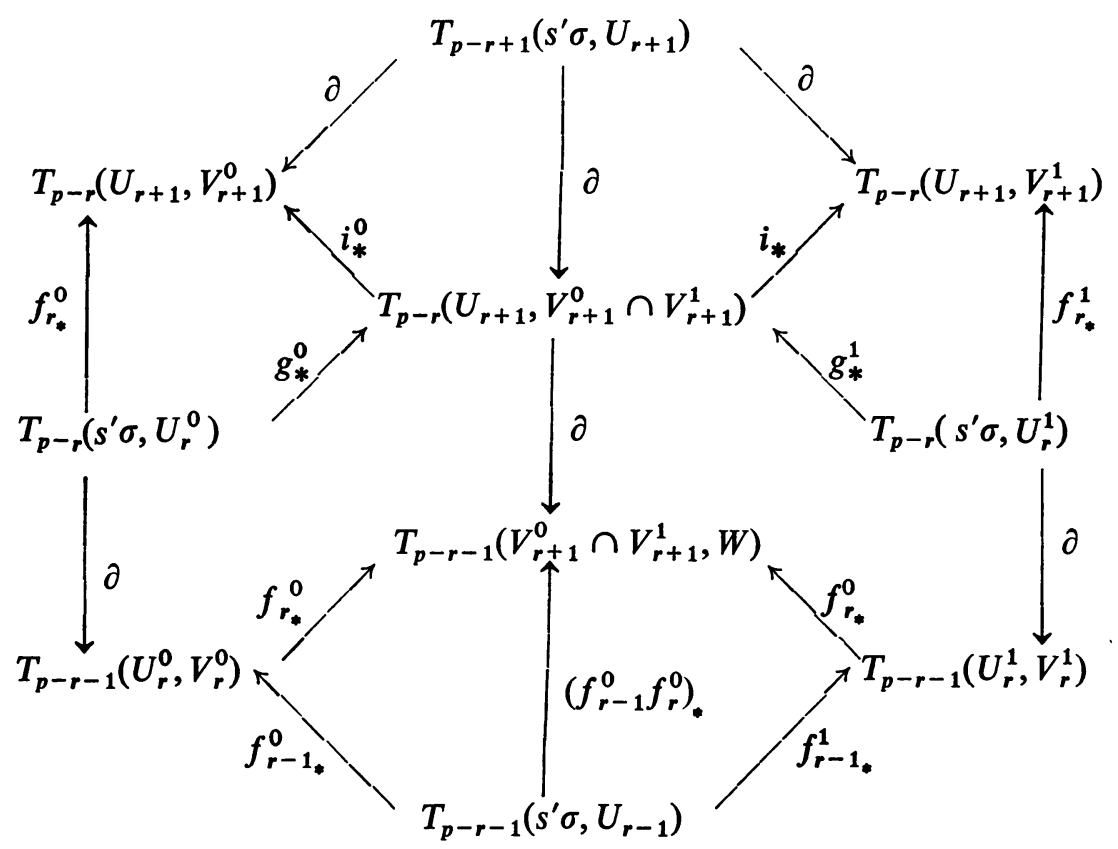

Excision-isotopy arguments show that all of the homomorphisms labeled $f_{s *}^{\alpha}$ $(\alpha=0,1, s=r-1, r)$ are isomorphisms, whence $\left(f_{r-1}^{0} f_{r}^{0}\right)_{*}=\left(f_{r-1}^{1} f_{r}^{1}\right)_{*}$ is an isomorphism. The composite $\partial \partial$ (going down the center) is trivial, for it can be factored, 


$$
\begin{gathered}
T_{p-r+1}\left(s^{\prime} \sigma, U_{r+1}\right) \stackrel{\partial}{\rightarrow} T_{p-r}\left(U_{r+1}, V_{r+1}^{0} \cap V_{r+1}^{1}\right) \\
\rightarrow T_{p-r}\left(s^{\prime} \sigma, V_{r+1}^{0} \cap V_{r+1}^{1}\right) \stackrel{\partial}{\rightarrow} T_{p-r-1}\left(V_{r+1}^{0} \cap V_{r+1}^{1}, W\right) .
\end{gathered}
$$

The diagonal sequences are exact, for they can be factored,

$$
\begin{aligned}
& \begin{array}{ll}
T_{p-r}\left(s^{\prime} \sigma, U_{r}^{0}\right) \stackrel{f_{r *}^{0}}{\longrightarrow} T_{p-r}\left(V_{r+1}^{1}, V_{r+1}^{0} \cap V_{r+1}^{1}\right) \rightarrow T_{p-r}\left(U_{r+1}, V_{r+1}^{0} \cap V_{r+1}^{1}\right) & \rightarrow T_{p-r}\left(U_{r+1}, V_{r+1}^{1}\right), \\
T_{p-r}\left(s^{\prime} \sigma, U_{r}^{1}\right) \stackrel{f_{*}^{1}}{\longrightarrow} T_{p-r}\left(V_{r+1}^{0}, V_{r+1}^{0} \cap V_{r+1}^{1}\right) \rightarrow T_{p-r}\left(U_{r+1}, V_{r+1}^{0} \cap V_{r+1}^{1}\right) & \rightarrow T_{p-r}\left(U_{r+1}, V_{r+1}^{0}\right),
\end{array} \\
& \text { and in each case the first homomorphism is an isomorphism. }
\end{aligned}
$$

It now follows from the Hexagonal Lemma [2, p. 38] that

$$
f_{r_{*}}^{0} \partial\left(f_{r_{*}}^{0}\right)^{-1} \partial=-f_{r_{*}}^{1} \partial\left(f_{r_{*}}^{1}\right)^{-1} \partial
$$

whence $\left(f_{r-1_{*}}^{0}\right)^{-1} \partial\left(f_{r_{*}}^{0}\right)^{-1} \partial=-\left(f_{r-1_{*}}^{1}\right)^{-1} \partial\left(f_{r_{*}}^{1}\right)^{-1} \partial$ and $\phi^{0}=-\phi^{1}$.

6. Proof of Theorem 4.1. If $X$ is an $n$-dimensional space with a triangulation $\mathscr{T}$, define a filtration $X_{-1}=\square, X_{0}, \cdots, X_{n}=X$ by setting

$$
X_{p}=\bigcup\left\{s^{\prime} \sigma: \operatorname{dim} \sigma \leqq p, \sigma \text { is a simplex of } \mathscr{T}\right\} .
$$

As usual, such a filtration together with a suitable functor (in this case the isology functor $\left\{T_{p}\right\}$ ) determines a spectral sequence $\left\{E_{p q}^{r}\right\}$ with the following characteristics.

1. $E_{p q}^{2}$ is the $p$ th homology group of the chain complex whose $p$ th chain group is

$$
T_{p+q}\left(X_{p}, X_{p-1}\right)
$$

and whose boundary operator is obtained from the isology sequence of the triple $\left(X_{p+1}, X_{p}, X_{p-1}\right)$.

2. If $\pi_{p, q}$ is the image of $T_{p+q}\left(X_{p}\right)$ in $T_{p+q}(X)$, then

$$
E_{p q}^{\infty} \approx \pi_{p q} / \pi_{p-1 . q 1}
$$

Thus in order to prove Theorem 4.1, it suffices to show that the chain complex

$$
\left\{T_{p+q}\left(X_{p}, X_{p-1}\right), \partial\right\}
$$

is isomorphic to $C(X ; q)$.

Lemma 6.1. The inclusion maps $s^{\prime} \sigma, \dot{s}^{\prime} \sigma \rightarrow X_{p}, X_{p-1}$ induce a direct sum decomposition,

$$
\sum\left\{T_{p+q}\left(s^{\prime} \sigma, \dot{s}^{\prime} \sigma\right): \operatorname{dim} \sigma=p\right\} \approx T_{p+q}\left(X_{p}, X_{p-1}\right) .
$$


Proof. Writing $\bar{\sigma}$ in the form

define

$$
\bar{\sigma}=\dot{\sigma} * b \sigma,
$$

$$
a_{0} \sigma=\left\{t x+(1-t) b \sigma: x \in \dot{\sigma}, \quad 0 \leqq t \leqq \frac{1}{2}\right\}
$$

Let

$$
a \sigma=\left(a_{0} \sigma * K(\sigma)\right) \backslash K(\sigma) \text { and } \dot{a} \sigma=a \sigma \cap \dot{s}^{\prime} \sigma .
$$

Then $i: a \sigma, \dot{a} \sigma \rightarrow s^{\prime} \sigma, \dot{s}^{\prime} \sigma$ is an excision. If

then $\bar{U} \subset X_{p-1}$ and

$$
U=X_{p} \backslash \bigcup\{a \sigma: \operatorname{dim} \sigma=p\}
$$

$$
\bigcup\{a \sigma, \dot{a} \sigma): \operatorname{dim} \sigma=p\} \rightarrow\left(X_{p}, X_{p-1}\right)
$$

is an excision. Since the sets $a \sigma$ are mutually disjoint, we have

$$
\sum T_{p+q}(a \sigma, \dot{a} \sigma) \approx T_{p+q}\left(X_{p}\left|U, X_{p-1}\right| U\right) .
$$

The result follows.

For each simplex $\sigma$ of $\mathscr{T}$, let us choose a flag. On the one hand, these choices determine a system of incidence numbers for $\mathscr{T}$. On the other hand, they determine isomorphisms

$$
\phi: T_{p+q}\left(s^{\prime} \sigma, \dot{s}^{\prime} \sigma\right) \rightarrow T_{q}(s \sigma) .
$$

Combining these isomorphisms with the isomorphisms of Lemma 6.1 yields isomorphisms

$$
\psi: T_{p+q}\left(X_{p}, X_{p-1}\right) \rightarrow C_{p}(X ; q) .
$$

It is a routine matter to verify that the diagram below is commutative, when $\tau$ is a face of $\sigma$.

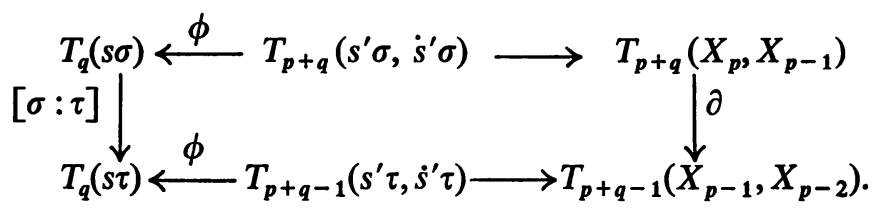

Thus $\psi$ commutes with the boundary homomorphisms. This establishes the desired isomorphism, and hence establishes Theorem 4.1.

7. Proof of Theorem 4.3. If $(X, A)$ is a pair with a triangulation $\mathscr{T}$, then

$$
A \subset A \cup X_{0} \subset \cdots \subset A \cup X_{n}
$$

defines a filtration on $X$. This filtration induces a spectral sequence $\left\{E_{p q}^{r}\right\}$ having the following properties.

1. $E_{q}^{2}$ is the homology of $\left\{T_{p+q}\left(X_{p} \cup A, X_{p-1} \cup A\right), \partial\right\}$. 
2. If $\pi_{p, q}$ is the image of $T_{p+q}\left(X_{p} \cup A, A\right)$ in $T_{p+q}(X, A)$, then

$$
E_{p q}^{\infty} \approx \pi_{p, q} / \pi_{p-1, q+1} \text {. }
$$

Thus in order to prove Theorem 4.3 , it suffices to show that $C(X, A ; q)$ is isomorphic to the complex whose $p$-dimensional group is

$$
T_{p+q}\left(X_{p} \cup A, X_{p-1} \cup A\right) .
$$

If $\sigma$ is a simplex of $\mathscr{T}$ and if $0<t \leqq 1$, let $u(\sigma, t)$ be the subset of $s^{\prime} \sigma$ consisting of the points

$$
v x+(1-v) y, \quad x \in K(\sigma), y \in \sigma, 0 \leqq v<t .
$$

Let

$$
\begin{aligned}
& X_{p}^{t}=\bigcup\{u(\sigma, t): \operatorname{dim} \sigma \leqq p\} \\
& A_{p}^{t}=A \cap X_{p}^{t}
\end{aligned}
$$

For any integers $p, q$ and for any $r, s, t, u \in I$ with $0<r \leqq s$ and $0<t \leqq u$, the inclusion map

$$
A_{p}^{r} \cup X_{q}^{t} \rightarrow A_{p}^{s} \cup X_{q}^{u}
$$

is an isotopy equivalence. To see this, pick a number $\alpha>0$ such that $\alpha s \leqq r$ and $\alpha u \leqq t$. Then

$$
v x+(1-v) y \rightarrow \alpha v x+(1-\alpha v) y, \quad x \in K(\sigma), y \in \sigma,
$$

defines an isotopy inverse to the inclusion map.

The inclusion maps induce isomorphisms,

$$
\begin{aligned}
T_{p+q}\left(A_{p}, A_{p-1}\right) & \approx T_{p+q}\left(A_{p} \cup X_{p-i}, X_{p-1}\right), \\
T_{p+q}\left(X_{p}, A_{p} \cup X_{p-1}\right) & \approx T_{p+q}\left(X_{p} \cup A, X_{p-1} \cup A\right) .
\end{aligned}
$$

To prove (7.1), factor the inclusion map,

$$
\left(A_{p}, A_{p-1}\right) \rightarrow\left(A_{p} \cup X_{p-1}^{t}, A_{p-1} \cup X_{p-1}^{t}\right) \rightarrow\left(A_{p} \cup X_{p-1}, X_{p-1}\right)
$$

with $t=\frac{1}{2}$. The first of these factors is an excision and it has already been noted that the second is an isotopy equivalence. To prove (7.2), observe that the following square of inclusion maps is commutative.

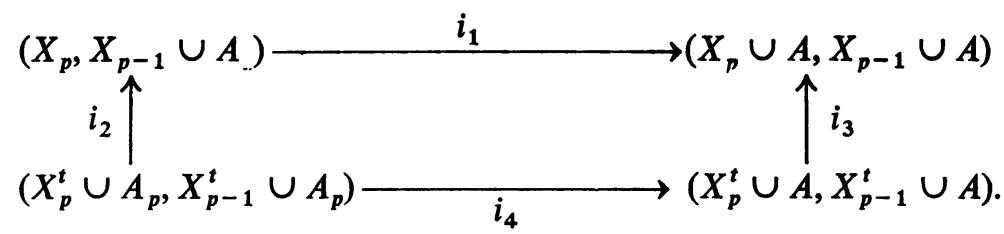


Again, $t=\frac{1}{2}$. The maps $i_{2}$ and $i_{3}$ are isotopy equivalences, and $i_{4}$ is an excision. Thus $i_{1_{*}}$ is an isomorphism.

Combining (7.1) with the isomorphism $\psi$ of $\S 6$, we obtain the commutative diagram,

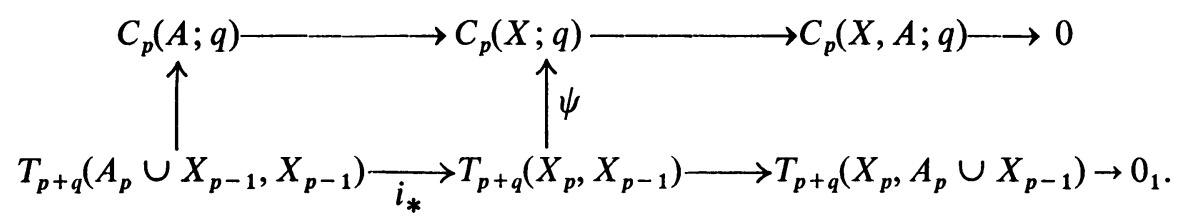

The two vertical homomorphisms are isomorphisms. The definition of $C_{p}(X, A ; q)$ insures that the upper sequence is exact. If $0_{1}$ is the kernel of

$$
i_{*}: T_{p+q-1}\left(A_{p} \cup X_{p-1}, X_{p-1}\right) \rightarrow T_{p+q-1}\left(X_{p}, X_{p-1}\right),
$$

then the lower sequence is exact. From the Monomorphism Postulate and the isomorphisms just described, it follows that $0_{1}$ is trivial. Thus

$$
C_{p}(X, A ; q) \approx T_{p+q}\left(X_{p}, A_{p} \cup X_{p-1}\right) \approx T_{p+q}\left(X_{p} \cup A, X_{p-1} \cup A\right) .
$$

The rest of the proof of 4.3 is routine.

8. Isology of one-complexes. One of the problems to which one may well hope to apply isology theory is the problem of classifying triangulable spaces up to isotopy type. One of the basic results in the corresponding area of homotopy theory is J. H. C. Whitehead's theorem that the homotopy functor distinguishes homotopy equivalences. The present section develops a primitive result of this nature for isotopy theory: there are isology theories which distinguish isotopy equivalences between 1-complexes.

All of the isology theories in this section are assumed to satisfy the Dimension Postulate $\{0\}$ and the Monomorphism Postulate. In addition they will be defined only on the category $\Gamma_{1}$ of embeddings of 1-dimensional triangulable pairs. These conventions will be used without further mention.

THEOREM 8.1. Every isology theory on the category of embeddings of 1-cones has a unique extension over $\Gamma_{1}$.

Proof. Corollary 4.4 guarantees the uniqueness of the extension. In the existence proof, the same symbol $\left\{T_{p}\right\}$ will be used for the extension as for the given isology theory on the cones.

Referring to the notation of $\$ 4$, note that if $(X, A) \in \Gamma_{1}$, then relative to a triangulation $\mathscr{T}$ of $(X, A)$, the chain complexes $C(X, A ; q)$ are defined and are trivial for $q \neq 0$. A slight elaboration of the usual proof for homology theory shows that a subdivision of $\mathscr{T}$ yields a chain equivalent complex. Since the Hauptvermutung holds on 1-complexes, 


$$
T_{p}(X, A)=H_{p}(C(X, A ; 0))
$$

is unambiguously defined. If the embedding

$$
f:(X, A) \rightarrow(Y, B)
$$

is in $\Gamma_{1}$, it may be made simplicial by inserting sufficiently many vertices in $X$ and in $Y$. The resulting chain transformation

$$
C(X, A ; 0) \rightarrow C(Y, B ; 0)
$$

defines $f_{*}=T_{p}(f)$. The functor that this defines is clearly an extension of the given isology theory on the 1-cones (whence it satisfies the Dimension Postulate $\{0\}$ and the Monomorphism Postulate) and only the demonstration that it is an isology theory remains.

Because the groups $T_{p}(X, A)$ arise from chain complexes, they automatically satisfy Axioms 1,2,3 and 4. The proof of the Excision Axiom is almost identical with the standard proof. Only the Isotopy Axiom remains.

Let $f_{t}:(X, A) \rightarrow(Y, B)$ be an isotopy. Let $X^{\prime}$ and $Y^{\prime}$ be the complements in $X$ and $Y$ (respectively) of the isolated vertices. For each simplex $\sigma$ of $X^{\prime}$ or $Y^{\prime}$ pick an embedding

$$
\lambda_{\sigma}: I \rightarrow s \sigma
$$

These induce

$$
\lambda_{\sigma^{*}}: T_{0}(I) \rightarrow T_{0}(s \sigma)
$$

and hence induce chain homomorphisms on the oriented chain groups,

$$
\begin{aligned}
\lambda: C_{p}\left(X^{\prime} ; T_{0}(I)\right) & \rightarrow C_{p}(X ; 0), \\
\lambda: C_{p}\left(Y^{\prime} ; T_{0}(I)\right) & \rightarrow C_{p}(Y ; 0) .
\end{aligned}
$$

Since $f_{t}$ is a homotopy, it induces a chain homotopy

with

$$
F^{\prime}: C_{p}\left(X^{\prime} ; T_{0}(I)\right) \rightarrow C_{p+1}\left(Y^{\prime} ; T_{0}(I)\right)
$$

$$
\partial F^{\prime}+F^{\prime} \partial=f_{1}-f_{0} .
$$

First note that $F^{\prime}$ is trivial except when $p=0$. Next, since $f_{t}$ carries $A$ into $B, F^{\prime}$ may be chosen to carry $C_{0}\left(A \cap X^{\prime} ; T_{0}(I)\right)$ into $C_{0}\left(B \cap Y^{\prime} ; T_{0}(I)\right)$. Finally, if $v$ is a vertex of $X$ which meets more than two 1-simplexes, then $f_{t}(v)$ must be independent of $t$. Thus $F^{\prime}$ may be chosen so as to be trivial on such vertices. Let

$$
G=\sum T_{0}(s v)
$$

the sum being over the vertices $v$ which meet just one or two 1 -simplexes. Then $G$ is a direct summand of $C_{0}(X ; 0)$ while the sum (not necessarily direct) of the 
subgroups $\operatorname{ker} F^{\prime}$ and $\lambda^{-1}(G)$ is $C_{0}\left(X^{\prime} ; T_{0}(I)\right)$. It follows that $F^{\prime}$ induces a chain homotopy

$$
F: C_{p}(X, A ; 0) \rightarrow C_{p+1}(Y, B ; 0)
$$

from $f_{1}$ to $f_{0}$. This establishes the Isotopy Axiom.

The 1-cones are easily classified. Each 1-cone consists of a vertex with a finite number $n$ of 1 -simplexes radiating from it. Note that $n=1$ and $n=2$ yield homeomorphic spaces. Aside from this case, the indexes $n=0,2,3, \cdots$ correspond to distinct isotopy types of spaces; the number $n$ will be called the order of the cone.

With the aid of this observation and Theorem 8.1, a large number of isology theories may be constructed. Let $G_{0} \subset G_{2} \subset G_{3} \subset \cdots$ be an increasing sequence of subgroups of some group $G$. If $A$ is a cone of order $m$, let

$$
T_{p}(A)= \begin{cases}G_{m} & \text { if } p=0 \\ 0 & \text { otherwise. }\end{cases}
$$

If $f: A \rightarrow B$ is an embedding of the cone $A$ in the cone $B$, let $T_{p}(f)$ be the inclusion homomorphism. Since the category of embeddings of 1-cones has no honest pairs in it, the functor $\left\{T_{p}\right\}$ is readily seen to be an isology functor. Theorem 8.1 now produces an extension of this functor over the embeddings of 1-dimensional triangulable pairs.

In a given triangulation, of a space or of a pair, each vertex is the point of junction of a number $n$ of 1 -simplexes. The number $n$ is called the order of the vertex. Note that the star of the vertex is a cone, and if $n \neq 1$, the order of the vertex is the same as the order of the star of the vertex. We say that the isology theory $\left\{T_{p}\right\}$ distinguishes cones if each embedding $f: A \rightarrow B$ of a cone $A$ of order $m$ in a cone $B$ of order $n>m$ has

$$
\operatorname{im}\left(f_{*}: T_{0}(A) \rightarrow T_{0}(B)\right)
$$

a nontrivial proper subgroup of $T_{0}(B)$.

THEOREM 8.2. If $\left\{T_{p}\right\}$ distinguishes cones and if $f: X \rightarrow Y$ is an embedding of 1-complexes such that

$$
f_{*}: T_{p}(X) \rightarrow T_{p}(Y)
$$

is an isomorphism for all $p$, then $f$ is isotopic to a homeomorphism of $X$ onto $Y$.

The proof is divided into four lemmas. Let us first observe that, because of the simple nature of 1-complexes, $X$ may be regarded as a subcomplex of $Y$. Let $G=T_{0}\left(I^{0}\right)$. Note that the hypotheses of 8.2 insure that $H_{p}(Y, X ; G)=0$ for all $p$. 
LEMMA 8.3. $Y$ has a triangulation such that $Y_{\mid} X$ has no vertices whose order in $Y$ is $=2$.

Proof. In a given triangulation of $Y$, let $v$ be a vertex, not in $X$, of order 2, and let $u, w$ be vertices such that the 1-simplexes containing $v$ are $\langle u, v\rangle$ and $\langle v, w\rangle$. Note that there is no simplex $\langle u, w\rangle$, for if there were, then

$$
g\langle u, v\rangle+g\langle v, w\rangle-g\langle u, w\rangle, \quad g \in G, g \neq 0,
$$

would be a nonbounding cycle in $C_{1}(Y, X ; G)$, contradicting the fact that $T_{1}(Y, X)=0$. Thus the broken line $\langle u, v\rangle \cup\langle v, w\rangle$ can be replaced by $\langle u, w\rangle$, eliminating the vertex $v$. The result follows by induction on the number of vertices of order 2 .

LEMMA 8.4. In the triangulation of Lemma 8.3, if $\sigma$ is a 1-simplex in $Y \backslash X$, then one of its vertices lies in $X$, and the other does not.

Proof. They can not both lie in $X$, since $T_{1}(Y, X)=0$, and at least one must lie in $X$, since $T_{0}(Y, X)=0$.

LEMMA 8.5. In any triangulation of $(Y, X)$, if $v$ is a vertex of $Y$ and if the order of $v$ is $n>2$, then $v$ is a vertex of $X$ and its order in $X$ is $n$.

Proof. Otherwise $T_{0}(Y, X)$ would have nonzero elements arising from the fact that

$$
T_{0}(X \cap s v) \rightarrow T_{0}(s v)
$$

was not an epimorphism.

In a similar way, one deduces the following.

LEMma 8.6. If $\sigma$ is a 1-simplex of $Y \backslash X$ and if $v$ is the vertex of $\sigma$ in $X$, then $v$ has order 2 in $Y$, and order 1 in $X$.

Proof of Theorem 8.2. Since $H_{0}(Y, X ; G)=0, Y \backslash X$ has no vertices of order 0. Also (Lemma 8.4) it has no vertices of order $n>2$. Assuming the triangulation of Lemma 8.3, one eliminates the vertices of order 2. Thus any point of $Y \backslash X$ is either a vertex of order 1 , or lies interior to a 1-simplex $\langle u, v\rangle$. Since $H_{1}(Y, X ; G)=0$, at most one of the vertices $u, v$ lies in $X$. Since $H_{0}(Y, X ; G)=0$, at least one lies in $X$. Let $v$ be the vertex in $X$. It follows from Lemma 8.5 that $v$ has order 2 in $Y$. Let $\langle v, w\rangle$ be the (unique) other 1-simplex which meets $v$, and note that $\langle v, w\rangle \subset X$ since $v$ has order 1 in $X$ (Lemma 8.5, again). The inclusion map of $\langle v, w\rangle$ in $\langle u, v\rangle \cup\langle v, w\rangle$ is isotopic to a homeomorphism onto, by means of an isotopy leaving $w$ fixed. This isotopy extends to an isotopy from the inclusion of $X$ in $X \cup\langle v, w\rangle$ to a homeomorphism onto. Since there are only finitely many 1-simplexes in $Y \mid X$, an iteration of this procedure yields an isotopy from the inclusion of $X$ in $Y$ to a homeomorphism of $X$ onto $Y$. 
A final remark about the topological type of 1-complexes. We have seen that an embedding $f: X \rightarrow Y$ of 1-dimensional triangulable spaces is equivalent to a homeomorphism if and only if it induces isomorphisms in a suitable isology theory, $\left\{T_{p}\right\}$. Let $C^{\prime \prime}(X)$ and $C^{\prime \prime}(Y)$ denote the quotient complexes $C^{\prime \prime}=\operatorname{coker} \lambda$ (the notation is that of Theorem 8.1). Then, comparing the exact homology sequences of these complexes we see that $f_{*}=T_{p}(f)$ is an isomorphism if and only if the following two conditions are met:

(1) $f_{*}: H_{*}\left(C^{\prime \prime}(X)\right) \approx H_{*}\left(C^{\prime \prime}(Y)\right)$, and

(2) $f_{*}: H_{*}\left(X ; T_{0}(I)\right) \approx H_{*}\left(Y ; T_{0}(I)\right)$.

Thus $X$ and $Y$ are homeomorphic if and only if there is an embedding $f$ of $X$ in $Y$ such that

(1) Each vertex of order $n>2$ in $X$ is carried by $f$ onto a vertex of the same order in $Y$, and

(2) $f$ induces isomorphisms in some nontrivial homology theory.

\section{REFERENCES}

1. J. W. Alexander, The combinatorial theory of complexes, Ann. of Math. (2) 31 (1930), 292-320.

2. S. Eilenberg and N. E. Steenrod, Foundations of algebraic topology, Princeton Univ. Press, Princeton, N. J., 1952.

3. S.-T. Hu, Isotopy invariants of topological spaces, Proc. Roy. Soc. Ser. A 255 (1960), 331-336.

4. I. M. James and J. H. C. Whitehead, Homology with zero coefficients, Quart. J. Math. Oxford (2) 9 (1958), 317-320.

5. G. W. Whitehead, Generalized homology theories, Trans. Amer. Math. Soc. 102 (1962), 227-283.

PuRdue University,

LAFAYETTE, INDIANA

NORTHWESTERN UNIVERSITY,

EVANSTON, ILLINOIS 